feelings of dryness at the base of the penis, around the testicles, under the foreskin, around the glans of the penis and in the anus. Discussions were also had about changes in the volume and consistency of seminal fluid released either prior to or during ejaculation. Another conversation theme revolved around how the symptoms they experienced affected their ability to engage in sexual intercourse. Forum users discussed how pain and dryness made sexual intercourse painful, resulting in them withdrawing from sexual activity indefinitely. Discussions were also had about the lack of information available to help understand and manage sexual dysfunction. Forum users discussed how feelings of embarrassment about the nature of the symptoms and the stigma of it being "a woman's disease" kept them from seeking medical assistance. Those who had sought medical assistance shared their belief that health professionals (HPs) were misdiagnosing their symptoms and were prescribing ineffective treatments. They also reported that their HPs were dismissive of symptoms and unwilling to assist further. Conclusion: Utilising conversations from an internet forum was an effective method to use to gain insight into some of the issues that males with SS experience with sexual functioning. The absence of accessible information and lack of support from HPs for males with SS is hinted at in this research. Future research should focus on identifying issues surrounding male sexual functioning as this will both guide future intervention strategies and allow HPs to publish material to better support males with SS. References:

[1] Braun, V., \& Clarke, V. (2006). Using thematic analysis in psychology. Qualitative Research in Psychology, 3(2), 77-101.

[2] van de Merwe, J. P. (2010). Sjögren's Syndrome: Information for patients and professionals. Rotterdam: Erasmus MC.

[3] White, M., \& Dorman, S. M. (2001). Receiving social support online: Implications for health education. Health Education Research, 16(6), 693-707.

Disclosure of Interests: None declared

DOI: 10.1136/annrheumdis-2020-eular.898

\section{SAT0634-HPR PATIENT-REPORTED OUTCOMES REGARDING TWO FORMS OF METHOTREXATE AUTOINJECTORS IN RHEUMATOID ARTHRITIS: AN INTERNATIONAL CROSS-OVER SURVEY.}

$\underline{\text { Y. Morvan }}^{1}$, J. D. Zeitoun ${ }^{2} .{ }^{1}$ Ipsos Healthcare, Paris, France; ${ }^{2}$ Saint-Antoine Hospital Assistance Publique Hôpitaux de Paris, Gastroenterology and Nutrition, Paris, France

Background: Several types of methotrexate (MTX) autoinjectors (AI) are currently marketed in rheumatoid arthritis (RA), yet comparative data are scarce. Objectives: Investigate respective perceptions of patients regarding two marketed forms of MTX Al via a survey conducted by a global market research company.

Methods: Patients with moderate to severe RA treated by one of the two forms of MTX Al were recruited. In each participating country (France, Ireland, United-Kingdom, Spain), the respective proportions of recruited patients were approximately aligned on local market shares. The two investigated devices were: A-Al/ The first MTX Al marketed in Europe: bigger size, with an activation button, without double injection sound-control, with a larger window; B-Al/ The second MTX Al commercialized in Europe: smaller and thinner size, without activation button, with double injection sound-control and a smaller window. Each patient was interviewed during 30 minutes on his or her satisfaction level with the currently used device. Then, they were presented the alternative Al and they could test it on skin-mimicking pads. After this step, the patients were interviewed on the alternative device.

Results: 100 patients were enrolled over one-month period (A-Al users, $n=65$; $B-A$ I users, $n=35$ ). Overall, $61 \%$ of $A$-Al users reported that B-Al was "better" or "much better" whereas $43 \%$ of B-Al users judged A-Al as "better" or "much better." When $\mathrm{B}-\mathrm{Al}$ users were asked to evaluate convenience elements of $\mathrm{A}-\mathrm{Al}$, recognition of injection ending, general design and ease of use were the indicators that were the most poorly judged $(60 \%, 54 \%$, and $46 \%$ respectively). When A-Al users were cross-tested for B-Al, injection mode, general feeling, and ease of use were the three items providing the greatest satisfaction $(80 \%, 77 \%$, and $75 \%$, respectively). When they were asked about the characteristics of their usual device, the button, the design of the device and discomfort associated with the injection were the most dissatisfactory elements $(30 \%, 31 \%, 34 \%$ respectively). Also, $73 \%$ of $\mathrm{A}-\mathrm{Al}$ users reported being interested in trying B-Al while $26 \%$ of B-Al users replied being so. Last, $95 \%$ of B-Al users declared being "very satisfied" or "totally satisfied", with ease of use and recognition of injection ending being the most attractive items ( $94 \%$ and $95 \%$ of high or full satisfaction respectively).

Conclusion: In this international cross-over survey, the newest autoinjector on the market, B-Al has shown to exhibit better reported outcomes with respect to ease of use and recognition of the end of the injection and other tested indicators.

Treatment of patients with RA should aim at the best care and must be based on a shared decision between the patient and the rheumatologist. ${ }^{1}$ In this scope, the shared decision making is permitted when the patient agrees with the choice of treatment (medication, administration route, device, etc.). Patient's involvement in decision making is assumed to lead to improvement in health outcomes such as a better adherence to the treatment. ${ }^{2}$

\section{References:}

[1] Smolen JS, Landewé R, Bijlsma J, et al EULAR recommendations for the management of rheumatoid arthritis with synthetic and biological disease-modifying antirheumatic drugs: 2016 update Annals of the Rheumatic Diseases 2017;76:960-977

[2] Nota,I.; Drossaert, C.H.; Taal,E.; Vonkeman, H.E.; van de Laar, M.A. Patient participation in decisions about disease modifying anti-rheumatic drugs: A cross-sectional survey. BMC Musculoskelet. Disord. 2014, 15, 333.

Disclosure of Interests: None declared

DOI: 10.1136/annrheumdis-2020-eular.3669

\section{SAT0635-HPR THE BURDEN OF SCLERODERMA FROM PATIENTS'} PERSPECTIVE

A. Opinc ${ }^{1}$, O. Brzezińska ${ }^{1}$, J. Makowska ${ }^{1} .{ }^{1}$ Medical University of Lodz, Department of Rheumatology, Łódź, Poland

Background: Scleroderma can significantly impair daily functioning by leading to multi-organ involvement and profound fatigue. Recent studies suggest that objective measures of the disease severity may not necessarily correspond with the patients' perception ${ }^{1}$.

Objectives: To evaluate the degree of disability and fatigue in patients with scleroderma, distinguish the most troublesome activities and assess which clinical symptoms had mostly impact on daily functioning.

Methods: An online questionnaire was created and distributed to English-speaking patients with scleroderma by means of online support groups. The questionnaire contained inquiries about demographical data, clinical symptoms as well as the Scleroderma Health Assessment Questionnaire (SHAQ, comprised of HAQ-DI and specific VAS scales) and Functional Assessment of Chronic Illness Therapy - Fatigue (FACIT-F). Standard and Alternative Disability Indexes (SDI and ADI) were counted based on answers in HAQ-DI part. Degrees of disability and fatigue, reflected by SDI, $\mathrm{ADI}$ and FACIT-F scores, were compared between subgroup of patients with and without particular clinical symptoms. Responses were analysed statistically.

Results: Questionnaire was completed by 85 responders (mean age $48,93 \pm 14,4$ $98,82 \%$ were female). Mean SDI was $1.09 \pm 0.66$ with $41.18 \%$ of patients fulfilling the criteria of mild to moderate difficulty $(\mathrm{SDI}<1), 50.59 \%$ classified as moderate to severe disability $(1 \leq \mathrm{SDI}<2)$ and $8.24 \%$ being severely or very severely disabled $(\mathrm{SDI} \geq 2)$. Mean value of $\mathrm{ADI}$ was $0.93 \pm 0.61 .56 .47 \%$ of responders needed aid of the caregivers while gripping/opening, $31.76 \%$ while reaching and $29.41 \%$ while running errands and chores. Most frequently used facilitating devices included devices for gripping/opening (32.94\%) and for maintaining hygiene (21.18\%). Mean value of overall disease severity was estimated by VAS as $52.06 \pm 0.66$. The mean values of VAS assessing the impact on daily living were respectively $48.16 \pm 29.28$ for pain related to the disease, $43.34 \pm 34.94$ for Raynaud's, $41.86 \pm 34.59$ for gastrointestinal problems, $31.60 \pm 31.38$ for breathing difficulties and $20.85 \pm 33.47$ for finger ulcers. Mean FACIT-F score was $23.62 \pm 11.88$ yet $71.76 \%$ of patients fulfilled the criteria of severe fatigue. Both SDI and ADI correlated with FACIT-F score (respectively $r=-0.5684, p<0.0001$ and $r=-0.5711, p<0.0001)$. SDI, ADI and FACIT-F correlated with VAS scores for overall disease severity, pain, gastrointestinal and breathing problems. Patients with symptoms such as arthralgia, muscle weakness, dysphagia, blurred vision, hair loss, unintentional weight loss and hoarseness were statistically more disabled and fatigued as compared to patients without such symptoms. Responders with skin ulcers/non-healing wounds (other than finger ulcers), Raynaud's or erythema/rash were more disabled than responders without such symptoms, yet no differences were observed in the degree of fatigue.

Conclusion: Majority of patients with scleroderma are moderately to severely disabled yet cope with the impairments with the widespread use of facilitat ing devices or aid of caregivers. Profound fatigue, observed in the majority of patients, may contribute to the high overall perception of disease severity. References:

[1] Jaeger VK, Distler O, Mauer B et al. Functional disability and its predictors in systemic sclerosis: a study from the DeSScipher project within the EUSTAR group. Rheumatology (Oxford). 2018 Mar 1;57(3):441-450. doi: 10.1093/ rheumatology/kex182

Disclosure of Interests: None declared

DOI: 10.1136/annrheumdis-2020-eular.3797

\section{SAT0636-HPR PATIENT EXPERIENCE WITH THE PRESCRIPTION, INFORMATION AND USE OF METHOTREXATE}

T. Oton ${ }^{1}$, L. Carmona ${ }^{1}$, J. L. Andréu Sánchez ${ }^{2} .{ }^{1}$ InMusc. Instituto de Salud Musculoesquelética, Madrid, Spain; ${ }^{2}$ Rheumatology Department. Hospital Universitario Puerta de Hierro-Majadahonda, Majadahonda, Spain

Background: Methotrexate (MTX) is currently a mainstream drug in the treatment of rheumatic diseases. However, the response to MTX is not universal and 